\title{
Analysis of 15 short tandem repeats reveals significant differences between the Arabian populations from Morocco and Syria
}

\author{
Louai Abdin $^{\mathrm{a}, \mathrm{b}}$, Ichiroh Shimada ${ }^{\mathrm{a}, \mathrm{c}}$, Bernd Brinkmann ${ }^{\mathrm{a}, *}$, Carsten Hohoff ${ }^{\mathrm{a}}$ \\ ${ }^{a}$ Institut für Rechtsmedizin, Universitätsklinikum Münster, Röntgenstrasse 23, D-48149 Münster, Germany \\ ${ }^{\mathrm{b}}$ Department of Legal Medicine, University of Tishreen, Latakia, Syria \\ ${ }^{\mathrm{c}}$ Department of Legal Medicine, Toyama Medical and Pharmaceutical University, Toyama, Japan
}

\begin{abstract}
The short tandem repeat (STR) systems D3S1358, TH01, D21S11, D18S51, Penta E, D5S818, D13S317, D7S820, D16S539, CSF1PO, Penta D, vWA, D8S1179, TPOX and FGA were studied in Arabian population samples from Morocco and Syria. No significant deviation from Hardy-Weinberg equilibrium could be observed in either preparation. Comparing the Moroccan and Syrian populations using the program $\mathrm{R} \times \mathrm{C}$, no similarity could be observed at all 15 loci. In the Moroccan and Syrian populations the matching probability is 1 in $1.4 \times 10^{17}$ and 1 in $2.6 \times 10^{17}$, respectively. Thus, the combination of these 15 STR loci is powerful tool for forensic identification in Arabian populations.
\end{abstract}

(C) 2003 Elsevier Science Ireland Ltd. All rights reserved.

Keywords: PowerPlex 16; Multiplex polymerase chain reaction; Morocco; Syria; Population genetics

\section{Introduction}

Short tandem repeat (STR) typing has become the state-of-the-art technique for forensic identification and paternity testing $[1,2]$. The aim of this work was to establish a STR database for the Arabian populations from Morocco and Syria, located at the very West and East of the Mediterranean Sea, respectively, and to check if they can be fused to a single Arabian database. Therefore, we have applied PowerPlex 16 System (Promega, Madison, WI) that amplifies the seven ENFSI STR loci as well as 13 CODIS STR loci in addition to the two pentanucleotide repeat loci, Penta E and Penta D, and the amelogenin locus for gender identification [3].

\footnotetext{
* Corresponding author. Fax: +49-251-835-5158.

E-mail address: brinkma@uni-muenster.de (B. Brinkmann).
}

In this study we present the allele frequencies and forensic efficiency values for the 15 STR loci in the Moroccan and Syrian populations and compare them.

\section{Materials and methods}

The population samples examined have already been described elsewhere [4,5]. DNA from the blood or saliva samples was extracted by the proteinase K/chelex-100 method [6] and amplified according to the recommendations of the manufacturer for the PowerPlex 16 System (Promega) except for using $12.5 \mu$ l polymerase chain reaction (PCR) volumes in a GeneAmp 9600 (Applied Biosystems, Foster City, CA). PCR products were separated by capillary gel electrophoresis on an ABI PRISM 310 Genetic Analyzer with laser-induced fluorescence (LIF) detection. Evaluation of the Hardy-Weinberg equilibrium and 
Table 1

Allele frequencies and forensic efficiency parameters for 15 STR loci in a Moroccan population $(n=138)$

\begin{tabular}{|c|c|c|c|c|c|c|c|c|c|c|c|c|c|c|c|}
\hline Allele & D3S1358 & TH01 & D21S11 & D18S51 & Penta E & D5S818 & D13S317 & D7S820 & D16S539 & CSF1PO & Penta D & vWA & D8S1179 & TPOX & FGA \\
\hline 2.2 & & & & & & & & & & & 0.02 & & & & \\
\hline 5 & & & & & 0.032 & & & & & & 0.004 & & & & \\
\hline 6 & & 0.168 & & & 0 & & & & & & 0 & & & 0.015 & \\
\hline 7 & & 0.193 & & & 0.143 & & & 0.004 & & & 0.012 & & & 0.019 & \\
\hline 8 & & 0.182 & & & 0.028 & 0.022 & 0.099 & 0.115 & 0.039 & 0.028 & 0.016 & & 0.004 & 0.404 & \\
\hline 9 & & 0.336 & & 0.008 & 0.032 & 0.033 & 0.047 & 0.133 & 0.098 & 0.02 & 0.159 & & 0.007 & 0.135 & \\
\hline 9.3 & & 0.117 & & 0 & 0 & 0 & 0 & 0 & 0 & 0 & 0 & & 0 & 0 & \\
\hline 10 & & 0.004 & & 0.004 & 0.103 & 0.069 & 0.044 & 0.311 & 0.051 & 0.276 & 0.134 & & 0.067 & 0.077 & \\
\hline 11 & & & & 0.004 & 0.155 & 0.281 & 0.318 & 0.2 & 0.285 & 0.311 & 0.236 & & 0.144 & 0.335 & \\
\hline 12 & & & & 0.146 & 0.179 & 0.372 & 0.339 & 0.204 & 0.313 & 0.339 & 0.122 & & 0.104 & 0.015 & \\
\hline 13 & & & & 0.122 & 0.119 & 0.212 & 0.109 & 0.033 & 0.168 & 0.024 & 0.211 & 0.004 & 0.252 & & \\
\hline 14 & 0.073 & & & 0.138 & 0.02 & 0.011 & 0.044 & & 0.047 & 0.004 & 0.077 & 0.134 & 0.244 & & \\
\hline 15 & 0.266 & & & 0.106 & 0.024 & & & & & & 0.004 & 0.145 & 0.144 & & \\
\hline 16 & 0.255 & & & 0.159 & 0.04 & & & & & & 0.004 & 0.236 & 0.03 & & \\
\hline 17 & 0.255 & & & 0.175 & 0.075 & & & & & & & 0.243 & 0.004 & & \\
\hline 18 & 0.131 & & & 0.061 & 0.032 & & & & & & & 0.159 & & & \\
\hline 19 & 0.015 & & & 0.041 & 0.008 & & & & & & & 0.054 & & & 0.071 \\
\hline 19.2 & 0 & & & 0 & 0 & & & & & & & 0 & & & 0.004 \\
\hline 20 & 0.004 & & & 0.028 & 0.004 & & & & & & & 0.025 & & & 0.098 \\
\hline 21 & & & & 0.008 & 0.008 & & & & & & & & & & 0.18 \\
\hline 22 & & & & & & & & & & & & & & & 0.173 \\
\hline 22.2 & & & & & & & & & & & & & & & 0.008 \\
\hline 23 & & & & & & & & & & & & & & & 0.18 \\
\hline 24 & & & & & & & & & & & & & & & 0.143 \\
\hline 24.2 & & & 0.004 & & & & & & & & & & & & 0 \\
\hline 25 & & & 0 & & & & & & & & & & & & 0.079 \\
\hline 26 & & & 0 & & & & & & & & & & & & 0.049 \\
\hline 27 & & & 0.007 & & & & & & & & & & & & 0.008 \\
\hline 28 & & & 0.134 & & & & & & & & & & & & 0.004 \\
\hline 29 & & & 0.239 & & & & & & & & & & & & 0.004 \\
\hline 29.2 & & & 0.007 & & & & & & & & & & & & \\
\hline 30 & & & 0.228 & & & & & & & & & & & & \\
\hline 30.2 & & & 0.015 & & & & & & & & & & & & \\
\hline 31 & & & 0.063 & & & & & & & & & & & & \\
\hline 31.2 & & & 0.131 & & & & & & & & & & & & \\
\hline 32 & & & 0.007 & & & & & & & & & & & & \\
\hline 32.2 & & & 0.086 & & & & & & & & & & & & \\
\hline 33 & & & 0.004 & & & & & & & & & & & & \\
\hline 33.2 & & & 0.063 & & & & & & & & & & & & \\
\hline 35 & & & 0.011 & & & & & & & & & & & & \\
\hline
\end{tabular}


Table 1 (continued)

\begin{tabular}{lccccccccccccccc}
\hline Allele & D3S1358 & TH01 & D21S11 & D18S51 & Penta E & D5S818 & D13S317 & D7S820 & D16S539 & CSF1PO & Penta D & vWA & D8S1179 & TPOX & FGA \\
\hline$n$ & 137 & 137 & 134 & 123 & 126 & 137 & 137 & 135 & 128 & 127 & 123 & 138 & 135 & 130 & 133 \\
H. obs & 0.781 & 0.766 & 0.858 & 0.878 & 0.857 & 0.693 & 0.737 & 0.748 & 0.766 & 0.756 & 0.78 & 0.71 & 0.793 & 0.785 & 0.857 \\
H. exp & 0.779 & 0.778 & 0.843 & 0.875 & 0.89 & 0.734 & 0.759 & 0.793 & 0.78 & 0.714 & 0.838 & 0.821 & 0.822 & 0.703 & 0.864 \\
HWE & 0.304 & 0.111 & 0.496 & 0.215 & 0.638 & 0.414 & 0.109 & 0.441 & 0.903 & 0.692 & 0.233 & 0.002 & 0.144 & 0.499 & 0.848 \\
PD & 0.907 & 0.907 & 0.949 & 0.962 & 0.973 & 0.884 & 0.896 & 0.925 & 0.917 & 0.849 & 0.944 & 0.937 & 0.938 & 0.833 & 0.962 \\
PE & 0.564 & 0.538 & 0.711 & 0.751 & 0.709 & 0.418 & 0.488 & 0.507 & 0.537 & 0.52 & 0.563 & 0.444 & 0.585 & 0.571 & 0.709 \\
PIC & 0.74 & 0.74 & 0.82 & 0.86 & 0.88 & 0.69 & 0.72 & 0.76 & 0.74 & 0.66 & 0.81 & 0.79 & 0.79 & 0.65 & 0.85 \\
\hline
\end{tabular}

${ }^{a}$ H. obs, observed heterozygosity; H. exp, expected heterozygosity (unbiased); HWE, exact test probability performed by conventional Monte Carlo method; PD, power of discrimination; PE, power of exclusion; PIC, polymorphism information content. 
Table 2

Allele frequencies and forensic efficiency parameters for 15 STR loci in a Syrian population $(n=121)^{\mathrm{a}}$

\begin{tabular}{|c|c|c|c|c|c|c|c|c|c|c|c|c|c|c|c|}
\hline Allele & D3S1358 & TH01 & D21S11 & D18S51 & Penta E & D5S818 & D13S317 & D7S820 & D16S539 & CSF1PO & Penta D & vWA & D8S1179 & TPOX & FGA \\
\hline 2.2 & & & & & & & & & & & 0.004 & & & & \\
\hline 5 & & & & & 0.073 & & & & & & 0.004 & & & & \\
\hline 6 & & 0.267 & & & 0.004 & & & & & & 0 & & & 0.004 & \\
\hline 7 & & 0.15 & & & 0.112 & & 0.004 & 0.021 & & & 0.004 & & & 0.004 & \\
\hline 8 & & 0.154 & & & 0.017 & 0.013 & 0.147 & 0.13 & 0.027 & 0.004 & 0.017 & & 0.008 & 0.45 & \\
\hline 9 & & 0.254 & & & 0 & 0.047 & 0.059 & 0.105 & 0.149 & 0.004 & 0.165 & & 0.013 & 0.21 & \\
\hline 9.3 & & 0.133 & & & 0 & 0 & 0 & 0.004 & 0 & 0 & 0 & & 0 & 0 & \\
\hline 10 & & 0.042 & & 0.004 & 0.078 & 0.056 & 0.055 & 0.319 & 0.135 & 0.279 & 0.165 & & 0.071 & 0.059 & \\
\hline 11 & & & & 0.022 & 0.138 & 0.333 & 0.332 & 0.273 & 0.23 & 0.274 & 0.152 & & 0.058 & 0.248 & \\
\hline 11.3 & & & & 0 & 0 & 0 & 0 & 0 & 0 & 0 & 0 & & 0.004 & 0 & \\
\hline 12 & & & & 0.159 & 0.185 & 0.316 & 0.252 & 0.13 & 0.279 & 0.381 & 0.178 & & 0.125 & 0.025 & \\
\hline 13 & 0.004 & & & 0.124 & 0.112 & 0.226 & 0.097 & 0.017 & 0.144 & 0.053 & 0.2 & 0.004 & 0.296 & & \\
\hline 14 & 0.025 & & & 0.208 & 0.034 & 0.009 & 0.055 & & 0.032 & 0.004 & 0.074 & 0.087 & 0.2 & & \\
\hline 15 & 0.277 & & & 0.137 & 0.056 & & & & 0.005 & & 0.022 & 0.091 & 0.179 & & \\
\hline 16 & 0.277 & & & 0.124 & 0.03 & & & & & & 0.013 & 0.269 & 0.042 & & \\
\hline 16.4 & 0 & & & 0 & 0.004 & & & & & & & 0 & 0 & & \\
\hline 17 & 0.231 & & & 0.084 & 0.047 & & & & & & & 0.331 & 0.004 & & \\
\hline 17.2 & 0 & & & 0.004 & 0 & & & & & & & 0 & & & \\
\hline 18 & 0.164 & & & 0.071 & 0.047 & & & & & & & 0.161 & & & 0.013 \\
\hline 19 & 0.021 & & & 0.035 & 0.026 & & & & & & & 0.037 & & & 0.071 \\
\hline 20 & & & & 0.013 & 0.026 & & & & & & & 0.021 & & & 0.05 \\
\hline 21 & & & & 0.009 & 0.009 & & & & & & & & & & 0.172 \\
\hline 21.2 & & & & 0 & & & & & & & & & & & 0.021 \\
\hline 22 & & & & 0.004 & & & & & & & & & & & 0.139 \\
\hline 22.2 & & & & & & & & & & & & & & & 0.017 \\
\hline 23 & & & & & & & & & & & & & & & 0.172 \\
\hline 24 & & & & & & & & & & & & & & & 0.239 \\
\hline 24.2 & & & & & & & & & & & & & & & 0.004 \\
\hline 25 & & & & & & & & & & & & & & & 0.08 \\
\hline 26 & & & 0.004 & & & & & & & & & & & & 0.013 \\
\hline 27 & & & 0.022 & & & & & & & & & & & & 0.008 \\
\hline 28 & & & 0.088 & & & & & & & & & & & & \\
\hline 29 & & & 0.219 & & & & & & & & & & & & \\
\hline 29.2 & & & 0.004 & & & & & & & & & & & & \\
\hline 30 & & & 0.25 & & & & & & & & & & & & \\
\hline 30.2 & & & 0.066 & & & & & & & & & & & & \\
\hline 31 & & & 0.048 & & & & & & & & & & & & \\
\hline 31.2 & & & 0.083 & & & & & & & & & & & & \\
\hline 32.2 & & & 0.127 & & & & & & & & & & & & \\
\hline 33 & & & 0.018 & & & & & & & & & & & & \\
\hline 33.2 & & & 0.061 & & & & & & & & & & & & \\
\hline
\end{tabular}




\begin{tabular}{|c|c|c|c|c|c|c|c|c|c|c|c|c|c|c|c|}
\hline Allele & D3S1358 & TH01 & D21S11 & D18S51 & Penta E & D5S818 & D13S317 & D7S820 & D16S539 & CSF1PO & Penta D & vWA & D8S1179 & TPOX & FGA \\
\hline 34 & & & 0.004 & & & & & & & & & & & & \\
\hline 35 & & & 0.004 & & & & & & & & & & & & \\
\hline$n$ & 119 & 120 & 114 & 113 & 116 & 117 & 119 & 119 & 111 & 113 & 115 & 121 & 120 & 119 & 119 \\
\hline H. obs & 0.731 & 0.825 & 0.754 & 0.858 & 0.897 & 0.709 & 0.723 & 0.756 & 0.775 & 0.673 & 0.774 & 0.835 & 0.817 & 0.664 & 0.815 \\
\hline H. exp & 0.768 & 0.802 & 0.851 & 0.872 & 0.903 & 0.735 & 0.789 & 0.781 & 0.81 & 0.703 & 0.848 & 0.778 & 0.818 & 0.691 & 0.853 \\
\hline HWE & 0.349 & 0.703 & 0.094 & 0.775 & 0.409 & 0.89 & 0.788 & 0.423 & 0.74 & 0.712 & 0.22 & 0.735 & 0.781 & 0.242 & 0.6 \\
\hline PD & 0.902 & 0.924 & 0.953 & 0.963 & 0.974 & 0.885 & 0.928 & 0.92 & 0.934 & 0.854 & 0.951 & 0.909 & 0.938 & 0.852 & 0.957 \\
\hline PE & 0.478 & 0.646 & 0.517 & 0.712 & 0.788 & 0.443 & 0.464 & 0.521 & 0.553 & 0.387 & 0.552 & 0.665 & 0.63 & 0.375 & 0.627 \\
\hline PIC & 0.72 & 0.77 & 0.83 & 0.85 & 0.89 & 0.69 & 0.76 & 0.74 & 0.78 & 0.64 & 0.82 & 0.74 & 0.79 & 0.64 & 0.83 \\
\hline
\end{tabular}

${ }^{a}$ H. obs, observed heterozygosity; H. exp, expected heterozygosity (unbiased); HWE, exact test probability performed by conventional Monte Carlo method; PD, power of discrimination; PE, power of exclusion; PIC, polymorphism information content.

Table 3

Comparison between the Moroccan and Syrian populations ${ }^{\mathrm{a}}$

\begin{tabular}{llllllllllllllll}
\hline & D3S1358 & TH01 & D21S11 & D18S51 & Penta E & D5S818 & D13S317 & D7S820 & D16S539 & CSF1PO & Penta D & vWA & D8S1179 & TPOX & FGA \\
\hline$P$ & $<10^{-6}$ & $<10^{-6}$ & $<10^{-6}$ & $<10^{-6}$ & $<10^{-6}$ & 0.0225 & $<10^{-6}$ & $<10^{-6}$ & $<10^{-6}$ & $<10^{-6}$ & $<10^{-6}$ & $<10^{-6}$ & $<10^{-6}$ & $<10^{-6}$ & $<10^{-6}$ \\
\hline
\end{tabular}

${ }^{\text {a }}$ Probability values were calculated with the program R $\times$ C (M.P. Miller, http://bioweb.usu.edu/mpmbio). 
Table 4

Interpopulation comparison between Syria, Morocco and Egypt ${ }^{\mathrm{a}}$

\begin{tabular}{lll}
\hline & Moroccan-Egyptian & Syrian-Egyptian \\
\hline D3S1358 & 0.00014 & 0.03338 \\
D21S11 & $<10^{-6}$ & $<10^{-6}$ \\
D18S51 & $<10^{-6}$ & $<10^{-6}$ \\
D5S818 & $<10^{-6}$ & $<10^{-6}$ \\
D13S317 & $<10^{-6}$ & $<10^{-6}$ \\
D7S820 & $<10^{-6}$ & 0.00552 \\
vWA & $<10^{-6}$ & $<10^{-6}$ \\
D8S1179 & $<10^{-6}$ & $<10^{-6}$ \\
FGA & $<10^{-6}$ & $<10^{-6}$
\end{tabular}

a $P$ values were calculated with the program $\mathrm{R} \times \mathrm{C}$. Egypt data from Ref. [7].

other forensic statistical parameters was done with the computer programs TFPGA (M.P. Miller) and PowerStats (Promega), respectively. The frequency profile comparisons between the Arabian populations were carried out using the program $\mathrm{R} \times \mathrm{C}$, that performed Fisher's Exact test on contingency tables through the use of Metropolis algorithm (M.P. Miller, http:// bioweb.usu.edu/mpmbio).

\section{Results and discussion}

No deviation from Hardy-Weinberg equilibrium could be observed except for vWA in the Moroccan population (Tables 1 and 2). The most informative loci were D18S51, Penta E and FGA in both populations. The combined PD and PE values of all 15 loci in the Moroccan population were 0.9999999999999999928 and 0.9999983, respectively, and those in the Syrian population were 0.99999999999999999962 and 0.9999973 , respectively.

The interpopulation comparisons between Morocco and Syria revealed significant differences in all 15 loci (Table 3). Thus, a common STR database for the two Arabian populations could not be established. Therefore, we have compared our data with the Egyptian population [7], that is geographically located in between. Significant differences were observed at all nine loci that could be analyzed (Table 4).

According to the statistical parameters the combined analysis of these 15 STR loci is a powerful tool for forensic identification and paternity testing in the Arabian populations.

\section{Acknowledgements}

The authors would like to thank B. Botterbrod, M. Eppink, K. Möller, M. Schürenkamp, U. Sibbing and $\mathrm{K}$. Waterkamp for their excellent technical assistance during the stay of L.A. and I.S. in Münster.

\section{References}

[1] Shimada I, Rand S, Brinkmann B, Hohoff C. Kurdish population data for 11 STR loci (ACTBP2, CSF1P0, FGA, TH01, TPOX, vWA, D3S1358, D5S818, D7S820, D13S317 and D21S11). Int J Legal Med 2002;116:301-303.

[2] Klintschar M, Al-Hammadi N, Reichenpfader B. Significant differences between Yemenite and Egyptian STR profiles and the influence on frequency estimations in Arabs. Int J Legal Med 2001;114:211-214.

[3] Shimada I, Brinkmann B, Tuyen NQ, Hohoff C. Allele frequency data for 16 STR loci in the Vietnamese population. Int J Legal Med 2002;116:246-248.

[4] Laszik A, Sotonyi P, Rand S, Hohoff C. Frequency data for the STR locus ACTBP2 (SE33) in eight populations. Int J Legal Med 2001;115:94-96.

[5] Abdin L, Dewa K, Rand S, Hohoff C, Brinkmann B. Analysis of 13 Y-chromosomal STRs in an Arab population sample from Syria. Prog. Forensic Genet 2003;9:319-322.

[6] Banaschak S, Rolf B, Brinkmann B. Influence of different staining techniques on the DNA analysis of histological sections. Int J Legal Med 2000;113:114-116.

[7] Klintschar M, Al-Hammadi N, Reichenpfader B. Population genetic studies on the tetrameric short tandem repeat loci D3S1358, vWA, FGA, D8S1179, D21S11, D18S51, D5S818, D13S317 and D7S820 in Egypt. Forensic Sci Int 1999;104:2331. 